\title{
Cross-Layer Design for Downlink Scheduling Combined with Call Admission Control in Wireless Networks
}

\author{
Manikandan Arunachalam ${ }^{1}$ and Balasubadra Kandasamy ${ }^{2}$ \\ ${ }^{1}$ Department of ECE, R.M.K. College of Engineering and Technology, Puduvoyal, Tiruvallur, Tamil Nadu 601206, India \\ ${ }^{2}$ Department of CSE, R.M.D. Engineering College, Kavaraipettai, Tiruvallur, Tamil Nadu 601206, India
}

Correspondence should be addressed to Manikandan Arunachalam; mani_ac18@yahoo.co.in

Received 14 November 2016; Revised 9 January 2017; Accepted 29 January 2017; Published 15 May 2017

Academic Editor: Paolo Barsocchi

Copyright ( 2017 Manikandan Arunachalam and Balasubadra Kandasamy. This is an open access article distributed under the Creative Commons Attribution License, which permits unrestricted use, distribution, and reproduction in any medium, provided the original work is properly cited.

\begin{abstract}
Scheduling with admission control is proposed based on the integration of the parameters of physical layer and MAC layer called cross-layer design. To achieve the throughput, resource allocation is carried out based on the adaptive modulation and coding (AMC) in the physical layer and scheduling in MAC layer with rate adaptation. When link adaptation is applied, the total energy consumed will be reduced, which will lead to the optimization. Here the physical layer and MAC layer parameters are jointly analyzed to achieve the cross-layer integration for the proposed problem. Based on this cross-layer integration the packet delay and throughput of the multiuser system are analyzed. All the simulations were carried out in $3 \mathrm{G}$ simulation environment like Qualnet and Network Simulator and results were presented.
\end{abstract}

\section{Introduction}

In multiuser communication the resource allocation in wireless networks always leads to the tradeoff between the available resources and quality of service (QoS). Current OSI layer architecture works well independently in error free networks. But it does not work well for error prone networks [1]. For example, if the problem occurs at transport layer, it will blindly apply the congestion control mechanism to resolve the problem without knowing its base. Hence the cross-layer integration is required to address these problems [1]. Therefore, physical layer and MAC layers are combined to achieve the cross-layer integration for the resource allocation and scheduling. To achieve the cross-layer optimization, here the scheduling is proposed based on the channel state information $[2,3]$; that is, the user will be selected with fair channel condition to transmit the information. By this way, it is possible to improve the spectral efficiency of the system. Channel state information will have more impact on higher layers when the scheduling is carried out. Also, it is a must to have a cross-layer scheduler for an AMC based wireless system to define a tradeoff between the throughput and capacity [4]. Here, the scheduling algorithm is required to allocate the resources among the users efficiently when they are in fair and worst channel states [5]. But when dynamic resource allocation is demanded, it is required to involve call admission control algorithm with scheduling as an integrated part [6-10]. Admission control will play a major role when the mobile users are admitted with random variation in capacity.

It is a must to incorporate the scheduling algorithm when multiple users share the single base station $[6,7]$. This optimal scheduling algorithm will help the user with best quality using adaptive modulation and coding. This scheduling will be considered as opportunistic service which will deploy the channel state information (CSI) indicated by the mobile users through the feedback channel $[8,9]$. Hence the problem arises to define the proper call admission control algorithm which employs this opportunistic scheduling to provide assured QoS for the users. In this paper, two types of users with applications were considered. They are CBR (constant bit rate) applications and guaranteed users. Voice call users belong to CBR applications which impinge their data rate as a fixed one. If the assured throughput is not offered, then the performance of CBR applications will fade rigorously. Hence, these users are required to allot with minimum guaranteed throughput. At the same time, it is waste of resources by 
providing additional throughput more than what they need actually. The guaranteed users always require the minimum throughput and some times more than that also. Video call users belong to guaranteed applications. By providing the additional throughput, the users can accommodate the video rate to a random value without buffering and decoding. In this case, it is a must to provide the minimum assured throughput for the tolerable performance and some more to improve the performance if possible [11]. But the algorithm proposed in [11] did not address problem with constraints raised by the users. Here the proposed call algorithm will address this issue by allocating the calls based on the signal to noise ratio (SNR) and available bandwidth when the users are admitted with different constraints. Also, the packet scheduling with admission control and resource allocation were treated as integrated parts. In this approach, each time, the scheduler prepares the list of packets to be transmitted and then the resource will be allocated based on the channel state information when the users are admitted with different constraints. Here optimization is defined in terms of restricting the list of packets to be transmitted and the users too. The proposed system is an example of simple crosslayer integration between the scheduling and the resource allocation in the MAC layer. The call admission control (CAC) algorithm and the scheduling have been implemented in MAC layer where the AMC is raised in physical layer to achieve the cross-layer integration. Finally, our results were compared with high speed downlink packet scheduling algorithm (HSDPA). The rest of the paper is organized as follows: Section 2 discusses the proposed system model; Section 3 deals with PHY layer specifications; Section 4 discusses call admission control algorithm; Section 5 deals with scheduling; Section 6 is about cross-layer design; and simulation results are discussed in Section 7 followed by conclusion.

\section{System Model}

Figure 1 shows the end-to-end connection between the transmitter and the receiver of the wireless link. A buffer is implemented in the base station, which works with firstin-first-out (FIFO) mode and the AMC is performed by AMC selector which is deployed in the transmitter side [12]. The type of modulation and the coding is detected by the estimator which is placed along with the receiver. The AMC selector then demodulates and placed the data in the buffer of the receiver. In the physical layer of wireless link different modes are adopted. Each mode has specific modulation technique with different code rates. Depending on the modulation mode in the transmitter, the estimator chooses the corresponding modulation format; then the AMC selector demodulates the data [13]. The mode can be fixed depending on coding rate and the modulation technique.

Coherent demodulation and maximum-likelihood (ML) decoding are employed at the receiver. The decoded bit streams are mapped to packets, which are pushed upwards to layers above the physical layer.

At the physical layer, frame by frame transmissions are carried out, where each frame contains a fixed number

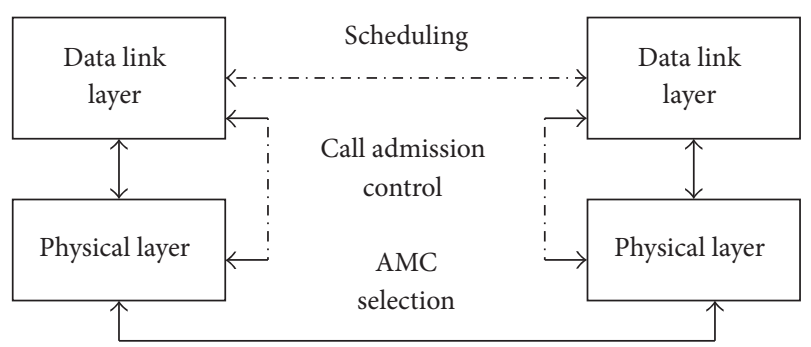

Adaptive modulation and coding (AMC)

Figure 1: Cross-layer architecture.

of symbols $\left(N_{s}\right)$. Each packet contains a fixed number of bits $\left(N_{b}\right)$, which include packet header, payload, and cyclic redundancy check (CRC) bits.

After modulation with mode $n$ of rate $R_{n}$, symbols are assigned to a block of $N_{b} / R_{n}$ symbols. Such multiple blocks constitute one frame to be transmitted at the physical layer.

\section{PHY Layer}

The system employs an AMC based on the fact that each user packet can be loaded with a considerable amount of bits. The AMC selection will be carried out by the AMC selector in the transmitter. PHY layer is supported with different modulation techniques such as QPSK, 16QAM, and 64QAM with convolutional encoding with different coding rates [3]. Here, data from the MAC layer fed back to PHY layer to achieve cross-layer design [4] (see Table 1).

In this AMC scheme, PER performance is prescribed based on the effective bandwidth by considering the transmission parameters in time varying channels adaptively such as IEEE $802.11 / 15 / 16$ and 3GPP/3GPP2 [6]. Assume that the number of transmission modes available is $N$. Also the SNR partition is assumed in the range of $N+1$ nonoverlapping intervals with the boundary of $\left\{\gamma_{n}\right\}_{n=0}^{N+1}$. In this case, mode $N$ will be selected if $\gamma \in\left[\gamma_{n}, \gamma_{n+1}\right]$ for $n=1,2, \ldots, N$.

The simplified BER expression in AWGN channel is given by (from [3])

$$
\operatorname{PER}(\gamma)= \begin{cases}1, & \text { if } 0<\gamma<\gamma_{p n}, \\ a_{n} \exp \left(-g_{n} \gamma\right), & \text { if } \gamma>\gamma_{p n} .\end{cases}
$$

Here $n$ is the index of transmission mode and $\gamma$ is the SNR. Parameters $a_{n}, g_{n}$, and $\gamma_{p n}$ in (1) are defined by mode and they are obtained by fixing (1) to the PER through simulations.

The region boundary can be set for the transmission mode $n$ in such a way that the minimum SNR is required to achieve the target packet error rate $P_{0}$. Now expression (1) can be modified as [3]

$$
\begin{aligned}
\gamma_{0} & =0, \\
\gamma_{n} & =\frac{1}{g_{n}} \ln \left(\frac{a_{n}}{P_{0}}\right), \quad n=1,2, \ldots, N, \\
\gamma_{N+1} & =+\infty .
\end{aligned}
$$


TABLE 1: Transmission modes with convolutionally coded modulation (the generator polynomial of the mother code is $g=[133,171]$ ).

\begin{tabular}{|c|c|c|c|c|c|}
\hline & Mode 1 & Mode 2 & Mode 3 & Mode 4 & Mode 5 \\
\hline Modulation & BPSK & QPSK & QPSK & 16-QAM & 64-QAM \\
\hline Coding rate $R_{c}$ & $1 / 2$ & $1 / 2$ & $3 / 4$ & $3 / 4$ & $3 / 4$ \\
\hline$R_{n}$ (bits/sym.) & 0.50 & 1.00 & 1.50 & 3.00 & 4.50 \\
\hline$a_{n}$ & 274.7229 & 90.2514 & 67.6181 & 53.3987 & 35.3508 \\
\hline$g_{n}$ & 7.9932 & 3.4998 & 1.6883 & 0.3756 & 0.0900 \\
\hline$\gamma_{p n}(\mathrm{~dB})$ & -1.5331 & 1.0942 & 3.9722 & 10.2488 & 15.9784 \\
\hline
\end{tabular}

With the above-mentioned boundaries we can verify that the AMC specified in the transmission mode assured that the PER is less than or equal to $P_{0}$. Also the AMC transmissions are designed to achieve maximum spectral efficiency, with the limited transmission modes based on the assumptions $[14,15]$.

\section{Call Admission Control (CAC)}

CAC is the process to regulate the traffic in wireless networks. This CAC algorithm decides whether to accept a call or not, in the network, while taking QoS constraints into account in a given cell capacity $[16,17]$. Thus, the aim of CAC scheme is to reduce new-call-blocking probability (CBP) and handoff call dropping probability (CDP) and to enhance the utilization of the cell capacity without wastage at the same time [18]. Without AMC, the scheduler allocates new frequency channel to the user when the communications channel quality fades. If the new channel already exists in the same base station, this belongs to intracell handoff. But with the help of AMC, the frequency channel with the faded condition can be reused $[19,20]$. This approach will help us to improve the effective utilization of the available resources. Here the joint effects of the AMC and CAC are analyzed based on the SNR and bandwidth.

In the proposed CAC algorithm, the QoS demands are considered based on the SNR and bandwidth. This algorithm employs the SNR to check for the availability of channels for new call admit by request. When a new call request arrives, it is classified into either CBR or guaranteed user. When the sufficient bandwidth is available then the CBR call is accepted and processed. Otherwise the base station calculates the SNR threshold $\left(\mathrm{SNR}_{\mathrm{th}}\right)$ for the requesting connection. The BS will update $\mathrm{SNR}_{\mathrm{th}}$ calculated for every new connection. If the measured $\mathrm{SNR}_{\mathrm{th}}$ is greater than or equal to the appropriate SNR and the bandwidth requirement is assured for every connection in the system, then the connection is admitted into the system. Sometimes with the marginal bandwidth, if the blocking probability is very low, then the call is accepted and the blocking probability will be updated. However, if the above-mentioned conditions are satisfied, but bandwidth availability is not assured, then connection requested will be accepted in the system through adaptive degradation. Otherwise, the call request is rejected. The proposed CAC algorithm was compared with the results proposed in $[16$, 17] through $3 \mathrm{G}$ simulation environment. The call blocking probability will be increased when $\mathrm{SNR}_{\text {th }}$ is fixed with a high value which leads to the signal quality and also reduces the utilization of networks. The $\mathrm{SNR}_{\mathrm{th}}$ value can be defined at the lowest level which maintains the blocking probability $P_{b}$ with the tolerable value. The blocking probability can be defined as

$$
P_{b}=\sum_{k=1}^{\infty} P_{o / k} P(k),
$$

where $P_{o / k}$ is the conditional outage probability and $P(k)$ is the probability that the $k$ users are admitted in a cell. $P_{o / k}=$ $1-\mathrm{Q}((\mathrm{SNR}-m) / \sigma)$, where $m$ is the mean and $\sigma$ is the standard deviation of SNR and $Q(x)=(1 / \sqrt{2 \pi}) \int_{x}^{\infty} e^{-t^{2}} d t$.

4.1. Admission Control Algorithm. $\mathrm{SNR}_{\text {th }}$ for the admission request should be calculated with the transmission error of wireless networks which is always below the acceptable threshold for the connection " $i$ ". Effective bit of the admission request is first calculated to obtain $\mathrm{SNR}_{\mathrm{th}}$. The effective bit rate is calculated as the minimum bandwidth required by the source of the traffic to the error threshold of transmission (Figure 2).

\section{Scheduling Algorithm}

In wireless networks, when the packets are needed to be scheduled with high security at physical layer, selection of the suitable link is the most complicated task. Also it is a must to ensure that the identified link should possess maximum throughput and quality of services for the users [21]. The scheduling process will be more complicated when the proposed CAC tends to the tolerable QoS failure in terms of delay and throughput. The role of the scheduler will be crucial in the part of maximizing the throughput and reducing the delay in all the admitted calls. In this case, EDF (earliest deadline first) will not be suitable to schedule the packets with the prescribed QoS. Earliest deadline packets are the prioritized packets to utilize the bandwidth without any delay. But in the case of real-time video transmission, it is mandatory to schedule some frames more than frames with earliest deadline to have the same flow. Another algorithm called weighted earlier deadline first (WEDF) is proposed in [22]. In this algorithm, the packets with longer distance will be prioritized even though the distance of the shorter distance packets is less than the longer distance packets. This algorithm will be able to keep the tradeoff between delay of long distance and short distance packets. But the main drawback of these algorithms is that they are less predicable and less controllable. 


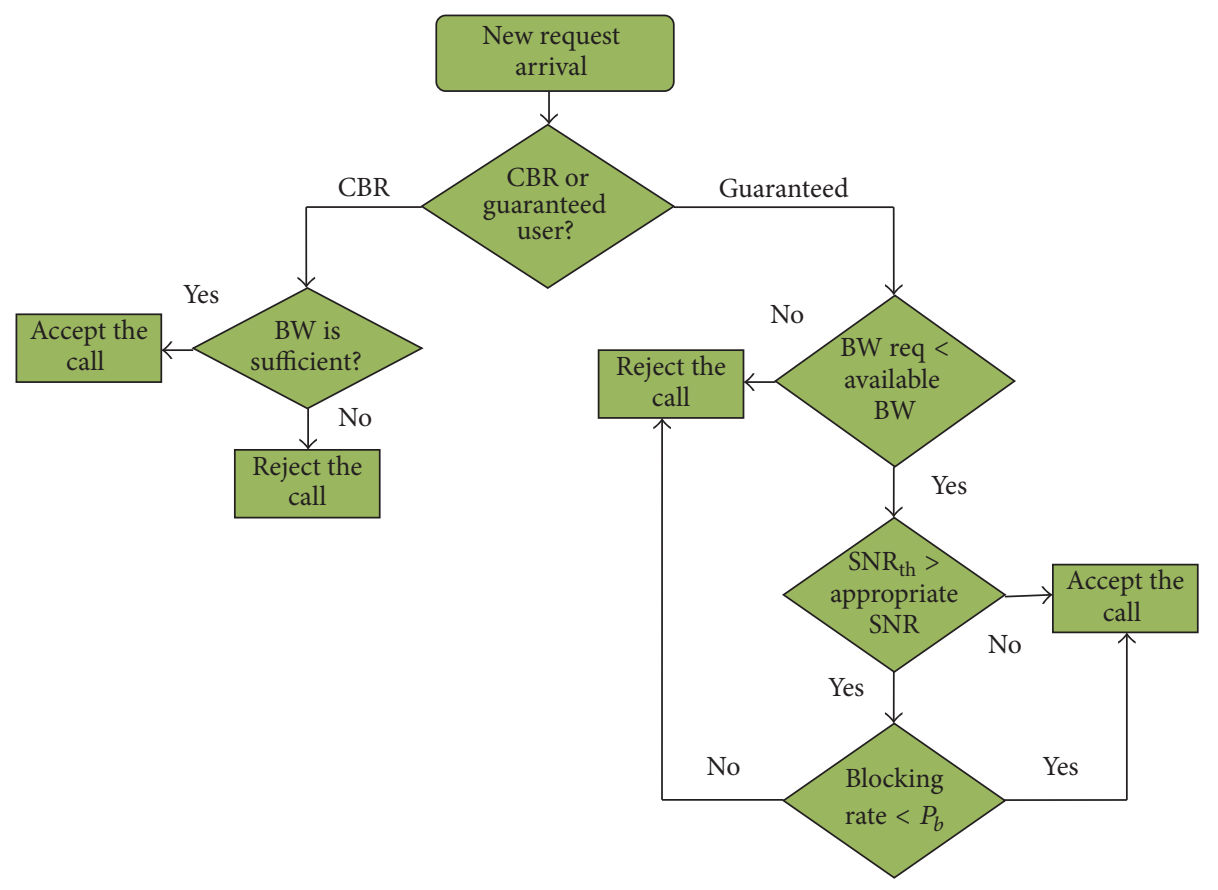

Figure 2: Call admission control flow.

Hence, the scheduling algorithm has to be designed in such a way to avoid the delay due to the previous frames and also based on the flow of the frame. Also, the tradeoff between the resource allocation and QoS profiles of services will be defined as a distribution of service classes " $n$ " numbers among " $k$ " number of resources. Based on the QoS parameters the services will be rated. An optimum method has to be identified to allocate the resources for the requested services. In our paper, the service classes of the QoS profiles are correlated with QoS index. The proposed scheduling algorithm determines the way to serve for each service class in the queue in order to assure the agreed QoS. While meeting this range of QoS, the call admission control (CAC) algorithm proposed in the previous section decides which service classes are to be accepted and which are to be rejected or it has to be held in delay call. Here the cross-layer approach of the CAC and scheduling algorithm will take the joint responsibility in a wide range to assure the QoS with major factors like queue length, QoS profile, and fairness. The scheduler will manage the tradeoff between maximizing the throughput and reducing the delay among the admitted calls at the same time. Packets received within the deadline will decide the amount of bandwidth to be utilized. The proposed scheduler emphasizes the area of the packets from one column to $N$ columns in the scheduler matrix as shown in Figure 3. While transmitting, the first packet of each row in the $N$ column is selected to be sent based on its delay. For each admission, the scheduler observes the total amount of data transmitted and error rate of the packets. Finally the potential packets in the $N$ columns will be selected to be sent based on the data recorded in the scheduler.

Here the packets with more demand will be considered to be scheduled so that those packets will receive the destination within the deadline fixed. Hence the packets were selected based on the bit error rate and the biasing can be reduced among the admitted flows.

\section{Cross-Layer Design}

Figure 1 shows the cross-layer design proposed in this paper. At PHY layer various modulation techniques like BPSK1/2, QPSK-1/2, QPSK-3/4, 16QAM-3/4, and 64QAM-3/4 have been used to observe their performance individually. In this paper, the proposed CAC algorithm and the scheduling have been implemented in the MAC layer. For CAC algorithm, the input parameter is SNR for all possible connections with the bandwidth available. CAC algorithm will make an optimized decision whether the call has to be accepted or rejected based on the input parameters given. The outputs call dropping probability, outage probability, and call blocking probability will feed back to the physical layer to select the optimum modulation and coding each and every time to assure the QoS [23]. The buffer size and delay requirements are the input parameters for the scheduler to reschedule the packets and share the bandwidth. Again the utilized bandwidth and queue fairness will be the output parameters of the scheduler which leads to selection of the modulation and coding at the physical layer. Therefore, the AMC technique will select the modulation and coding dynamically and the scheduler will decide the bandwidth allocation along with the AMC [23].

\section{Results and Discussions}

The numerical computations and simulations were accomplished in $3 \mathrm{G}$ wireless systems. The following assumptions 


\begin{tabular}{|c|c|c|c|c|c|}
\cline { 2 - 6 } \multicolumn{1}{c|}{} & Column 1 & Column 1 & Column 1 & $\ldots$ & Column N \\
\hline Row 1 & & & & & \\
\hline Row 1 & & & & & \\
\hline$\vdots$ & & & & & \\
\hline Row N & & & & & \\
\hline
\end{tabular}

FIgURE 3: Scheduler table.

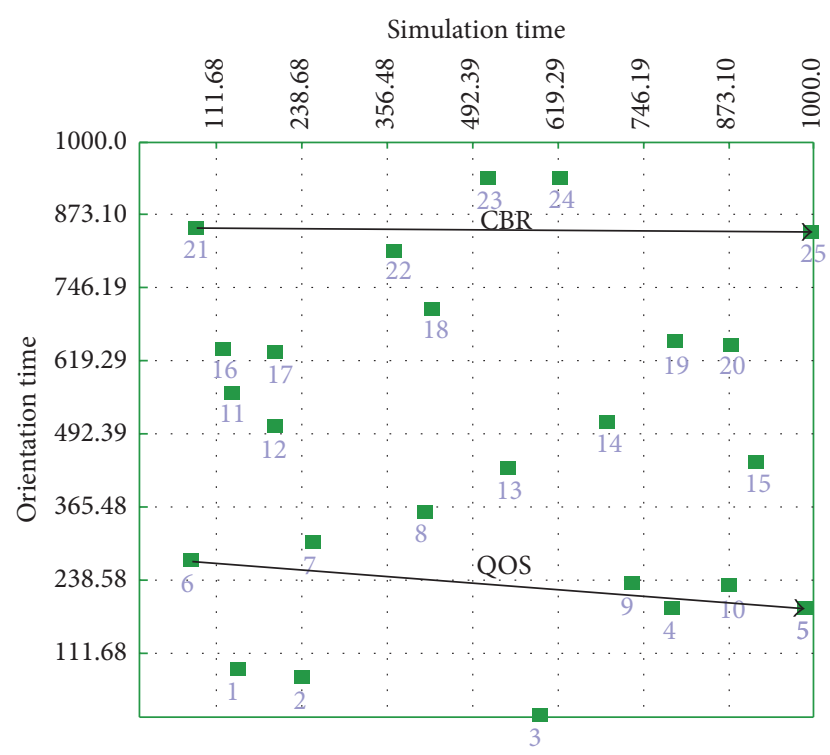

FIgURE 4: Initialization of nodes with CBR traffic.

were made during the simulations in CAC algorithm. Each cell has 30 channels. The new call arrivals in each cell follow a Poisson distribution. Each admitted call needs only one channel for service [24]. Call holding time exponentially distributed with mean of $200 \mathrm{~s}$.

In this section, the performance of our proposed algorithm has been evaluated in terms of throughput, call dropping probability, call blocking probability, and end-toend delay of users. Initially the simulation was started with 25 nodes and they are randomly placed in simulator grid. Figure 3 shows that how the nodes are initiated and placed in a grid with design mode. Later the simulation started with CBR traffic to measure the parameters like end-to-end delay and throughput is shown in Figure 5. The size of the window assumed at the base station is 100 packets. Scope graph has been captured for every 100 seconds. Figure 4 shows how the transmission can be initiated with 25 nodes in constant bit rate (CBR) traffic. Figure 5 shows how the nodes are communicating with others with minimum mobility.

In Figures 6 and 7, we can see that with increasing number of users the call dropping probability and call blocking probability are also increased. This is because of the increasing load in the network while admitting a more number of users. Figure 6 shows the comparison with algorithm proposed in $[16,17]$ and with CBR traffic. Results show that

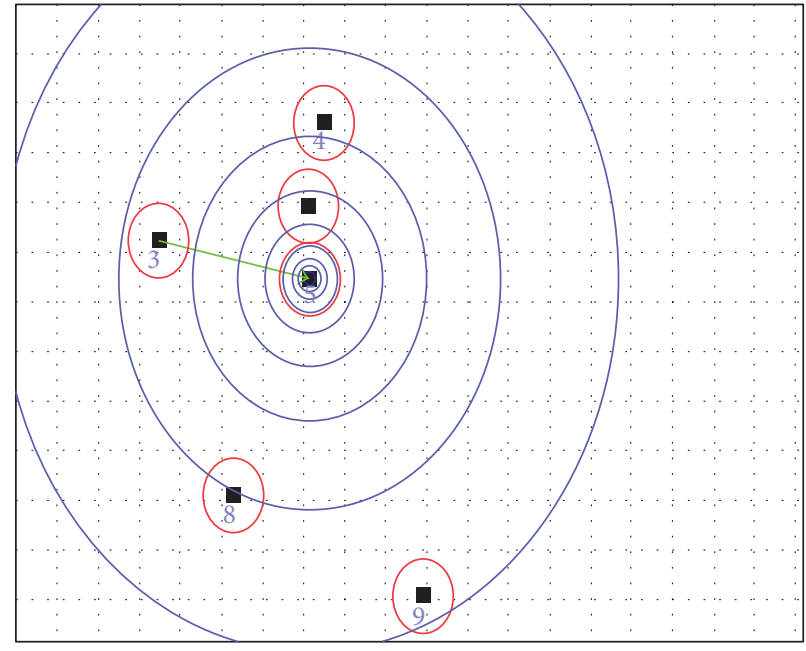

Figure 5: Mobility and data transmission between nodes.

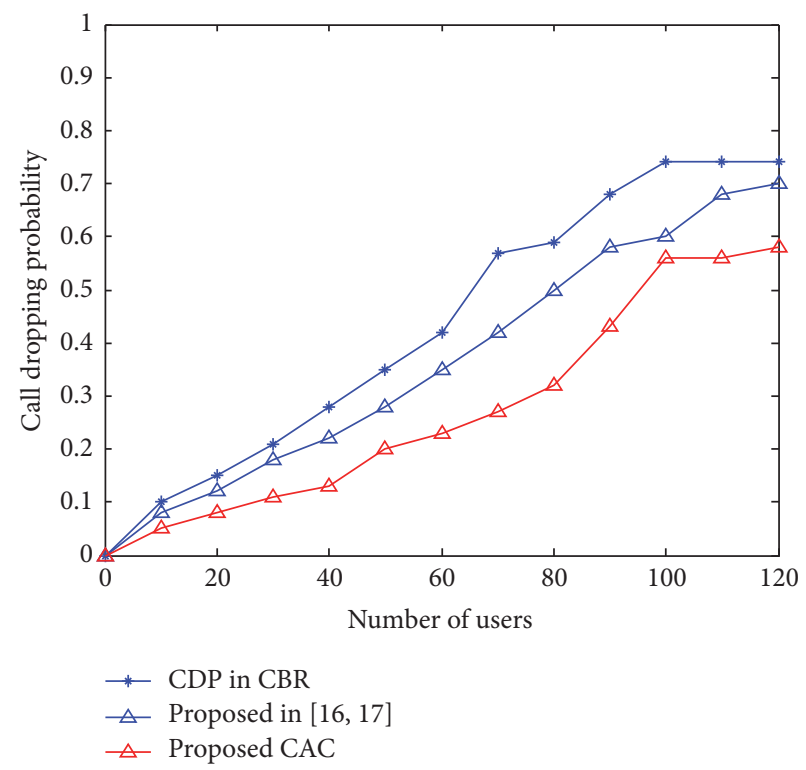

FIGURE 6: Number of users versus call blocking probability.

the proposed algorithm possesses least dropping probability when compared to the other algorithms.

It clearly shows that the proposed CAC scheme reduces the call blocking probability and is guaranteed compared to the solution. Similarly, Figure 7 shows the call dropping probability comparison of CBR traffic with our proposed algorithm. Here also, it clearly states that the dropping probability is far better when compared to the CBR traffic. From Figure 8 we can see that more number of calls can be admitted during the process, since the service rate of the proposed CAC algorithm by considering SNR is more efficient than the other algorithms. Because the single call will utilize very small fraction of the system resources, the remaining resources can be effectively distributed to other calls also. It is possible only when the call admission control admits the calls based on the SNR. 


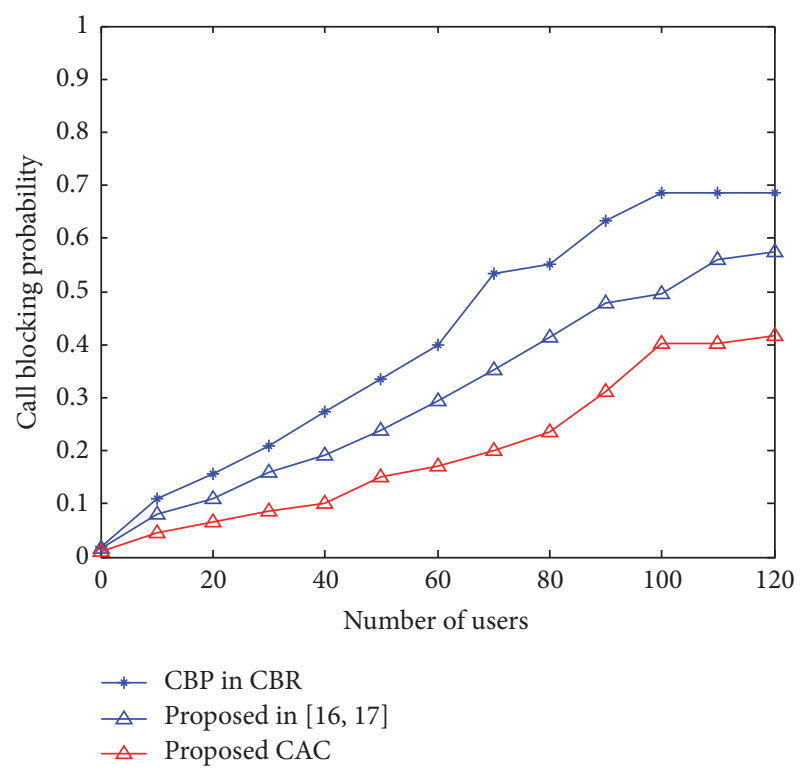

FIgURE 7: Number of users versus call dropping probability.

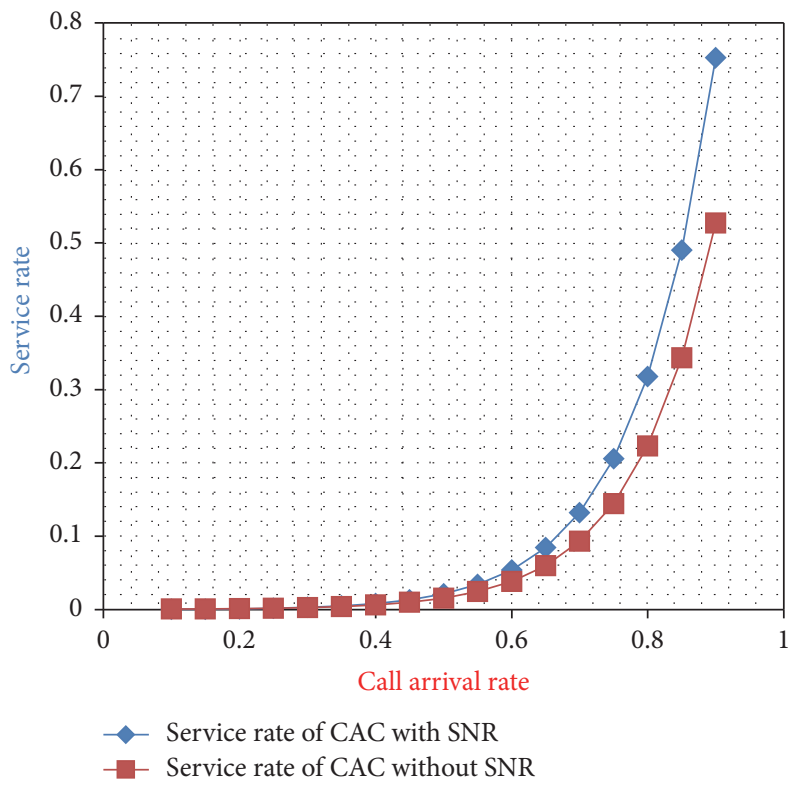

FIGURE 8: Service rate of the proposed CAC.

The impact of outage probability is described in Figure 10. Here we can see that, with increasing outage probability, the admission rate of the CBR and proposed CAC will also be increasing. The average admission rate of the proposed CAC algorithm is much better than that of the traffic with CBR. When more numbers of calls are requested to admit, the delay will be increased in the service to the existing calls. This can be justified from Figure 9. Even though the delay is comparatively high, our proposed algorithm pulls down the delay with number of users when compared with CBR traffic.

The throughput of the system is measured as the ratio of the number of bits received successfully over the simulation time. It will be calculated as bits per second. Figure 11 shows

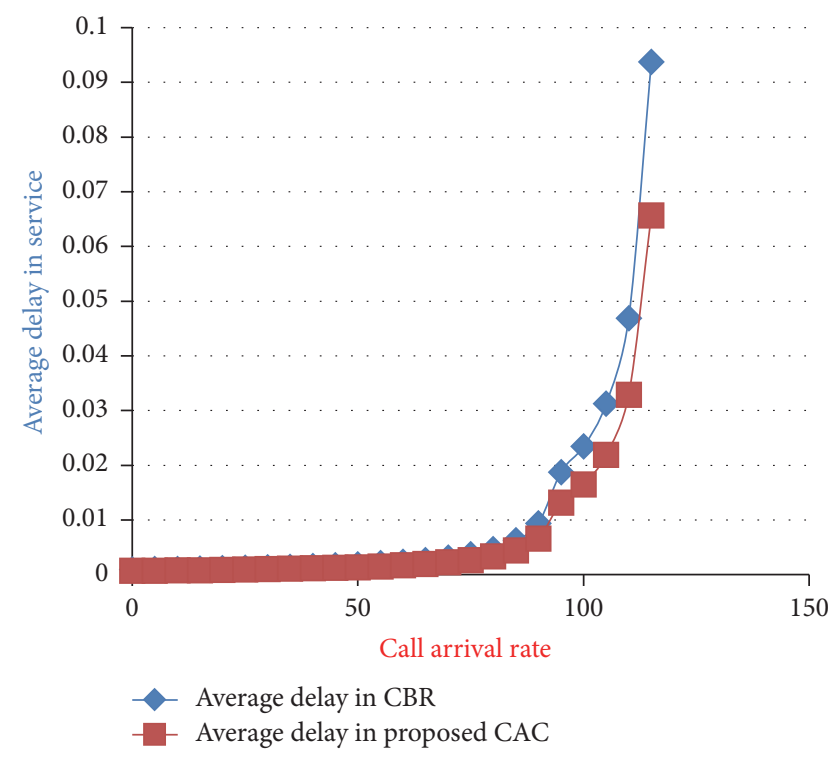

FIGURE 9: Average delay in service of the proposed CAC.

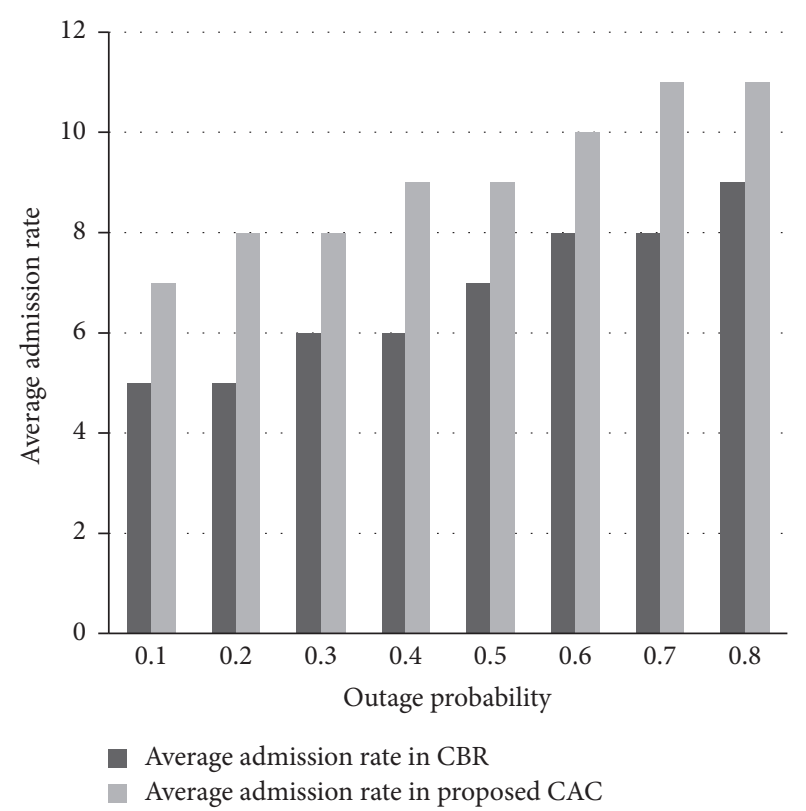

FIGURE 10: Outage probability of CAC.

the comparison of the system throughput in EDF, WEDF, and the proposed scheduling algorithms based on the number of users. The proposed cross-layer scheduling algorithm can server better than the other scheduling algorithms when the number of users is more. To serve more number of users, all the available resources are used effectively with smooth blocking probability.

Similarly the packet loss rate also will fall below $8 \%$ in the proposed cross-layer scheduling when it is more than $13 \%$ in EDF and WEDF. From Figure 12, we can see that the packet loss rate is much comparable with the existing algorithm. 


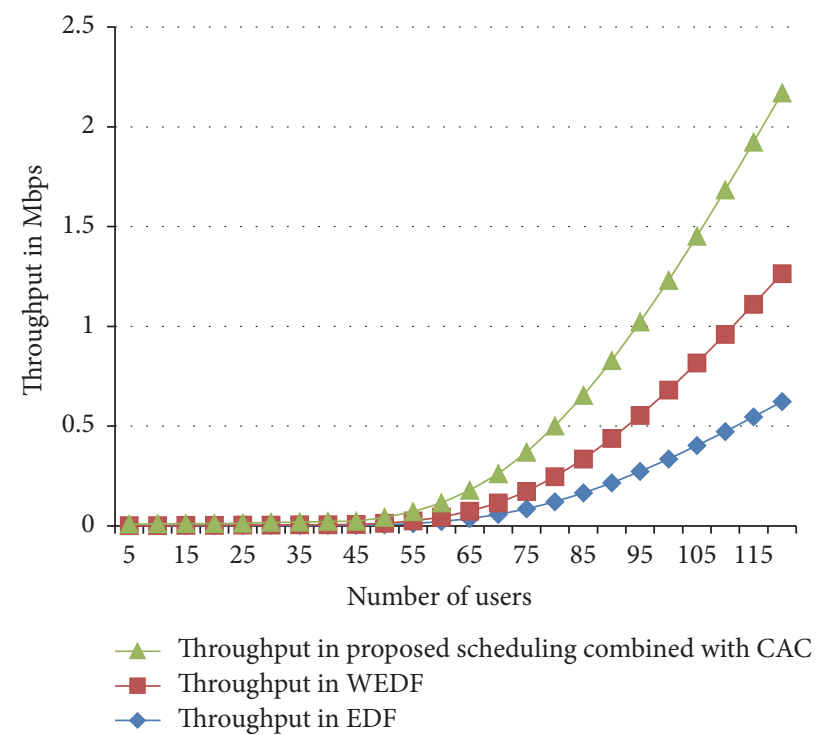

FIGURE 11: Throughput of the proposed cross-layer scheduling.

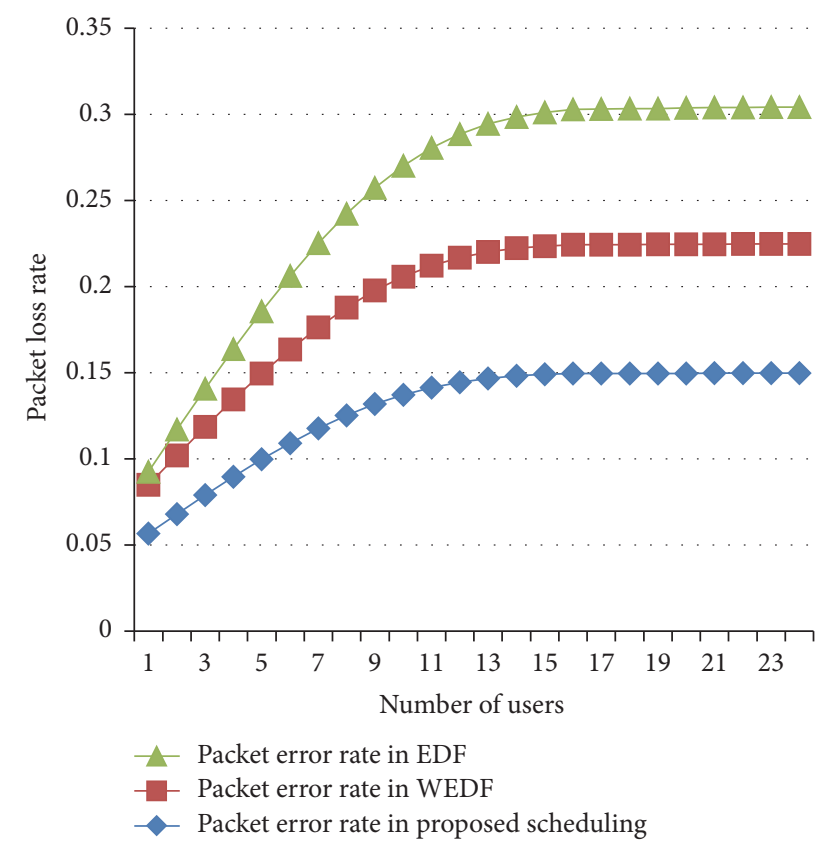

FIGURE 12: Packet loss rate of the proposed cross-layer scheduling.

\section{Conclusion}

In this paper, a novel cross-layer scheduling scheme with call admission control was studied under multiuser communication. Also the cross-layer architecture was developed with adaptive modulation and coding PHY layer and the scheduling MAC was carried out in the study of the effect of blocking probability, dropping probability, and outage probability in multiuser communication systems. The SNR based CAC with scheduling was analyzed to admit maximum number of calls with efficient usage of SNR. Every time, scheduler determines the optimum SNR to select the appropriate modulation and coding. The joint effect of the CAC and scheduling algorithms was analyzed and also found to be more efficient than the other algorithms in terms of QoS.

\section{Conflicts of Interest}

The authors declare that they have no conflicts of interest.

\section{References}

[1] B. Fu, Y. Xiao, H. Deng, and H. Zeng, "A survey of cross-layer designs in wireless networks," IEEE Communications Surveys \& Tutorials, vol. 16, no. 1, 2014.

[2] C. Manikandan, S. Bhashyam, and S. Rajesh, "Cross-layer scheduling with infrequent channel and queue measurements," IEEE Transactions on Wireless Communications, vol. 8, no. 12, pp. 5737-5742, 2009.

[3] Q. Liu, X. Wang, and G. B. Giannakis, "A cross-layer scheduling algorithm with QoS support in wireless networks," IEEE Transactions on Vehicular Technology, vol. 55, no. 3, pp. 839-847, 2006.

[4] A. Manikandan and K. Balasubadra, "Adaptive modulation \& coding for cross layer design - a case study," International Journal of Applied Engineering Research, vol. 9, no. 23, pp. 20255-20266, 2014.

[5] E. Kartsakli, J. Alonso-Zárate, L. Alonso, and C. Verikoukis, "Cross-layer scheduling with QoS support over a distributed queuing MAC for wireless LANs," Mobile Networks and Applications, vol. 14, no. 6, pp. 709-724, 2009.

[6] A. Haider and R. Harris, "A novel proportional fair scheduling algorithm for HSDPA in UMTS networks," in Proceedings of the 2nd International Conference on Wireless Broadband and Ultra Wideband Communications (AusWireless '07), pp. 43-50, Sydney, NSW, Australia, August 2007.

[7] B. Hamdaoui and P. Ramanathan, "A cross-layer admission control framework for wireless ad-hoc networks using multiple antennas," IEEE Transactions on Wireless Communications, vol. 6, no. 11, pp. 4014-4024, 2007.

[8] Y.-H. Tseng, E. H.-K. Wu, and G.-H. Chen, "An admission control scheme based on online measurement for VBR video streams over wireless home networks," IEEE Transactions on Multimedia, vol. 10, no. 3, pp. 470-479, 2008.

[9] Y. Zhang, "Call admission control in wireless networks over Rayleigh fading channel," in Proceedings of the 2007 IEEE Wireless Communications and Networking Conference (WCNC '07), pp. 3032-3036, Kowloon, China, March 2007.

[10] C. W. Leong, W. Zhuang, Y. Cheng, and L. Wang, "Optimal resource allocation and adaptive call admission control for voice/data integrated cellular networks," IEEE Transactions on Vehicular Technology, vol. 55, no. 2, pp. 654-669, 2006.

[11] L. Wang and W. Zhuang, "A call admission control scheme for packet data in CDMA cellular communications," IEEE Transactions on Wireless Communications, vol. 5, no. 2, pp. 406416, 2006.

[12] W. Li, "Overview of fine granularity scalability in MPEG-4 video standard," IEEE Transactions on Circuits and Systems for Video Technology, vol. 11, no. 3, pp. 301-317, 2001.

[13] A. Manikandan, S. Kamalakannan, B. B. Kumar, and S. V. Ramalingam, "Cross layer analysis of queuing with AMC through GMSK modulation over wireless links," in Proceedings of the 16th International Conference on Advanced Computing and 
Communications (ADCOM '08), pp. 260-264, Chennai, India, December 2008.

[14] A. Manikandan, A. K. Balasubadra, M. Vigneshwaran, T. S. Rakesh, and O. G. Ramprasad, "Cross layer analysis over fading channels," in Proceedings of the International Conference on Devices and Communications (ICDeCom '11), Mesra, India, February 2011.

[15] Q. Liu, S. Zhou, and G. B. Giannakis, "TCP performance in wireless access with adaptive modulation and coding," in Proceedings of the IEEE International Conference on Communications, pp. 3989-3993, 2004.

[16] 3GPP, "Physical layer aspects of utra high speed downlink packet access (release 4)," 3GPP TR 25.848 V4.0.0, 2001.

[17] M.-S. Alouini and A. J. Goldsmith, "Adaptive modulation over Nakagami fading channels," Wireless Personal Communications, vol. 13, no. 1, pp. 119-143, 2000.

[18] K. K. Radhia, M. K. Ben Ali, and L. Kammoun, "Allocation algorithm based on CAC scheme for LTE network," International Journal of Computer Science and Network Security, vol. 16, no. 6, pp. 140-150, 2016.

[19] D. M. Soleymani and V. TabaTabaVakili, "Improvement of system capacity using different frequency reuse and HARQ and AMC in IEEE 802.16 OFDMA networks," in Proceedings of the IAENG International Conference on Electrical Engineering (ICEE '12), Hong Kong, March 2012.

[20] M. Khabazian, O. Kubbar, and H. Hassanein, "Call admission control with resource reservation for multi-service OFDM networks," in Proceedings of the International Conference on Computing, Networking and Communications (ICNC '12), pp. 781-785, Maui, Hawaii, USA, February 2012.

[21] J. Wang, P. Huang, X. Wang, and Y. Yang, "Cross-layer scheduling for physical layer secrecy and queue stability in a multi-user system," in Proceedings of the Globecom Workshops (GC Wkshps '13), Atlanta, Ga, USA, December 2013.

[22] M. Oktay and H. A. Mantar, "A distance-aware scheduler for real-time applications in 802.16 Wimax networks," in Proceedings of the 1st International Conference on Networked Digital Technologies (NDT '09), pp. 435-440, Ostrava, Czech Republic, July 2009.

[23] R. Bruno, A. Masaracchia, and A. Passarella, "Robust Adaptive Modulation and Coding (AMC) selection in LTE systems using reinforcement learning," in Proceedings of the 80th IEEE Vehicular Technology Conference (VTC-Fall '14), pp. 1-6, Vancouver, BC, Canada, September 2014.

[24] H. Zeng, Y. Fang, and I. Chlamtac, "Call blocking performance study for PCS networks under more realistic mobility assumptions," Telecommunication Systems, vol. 19, no. 2, pp. 125-146, 2002. 


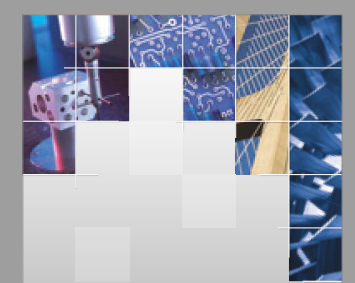

\section{Enfincering}
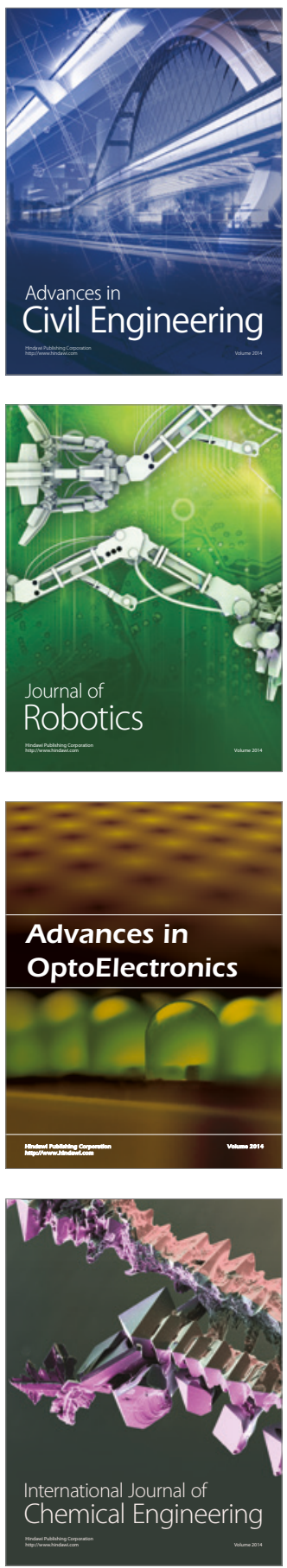

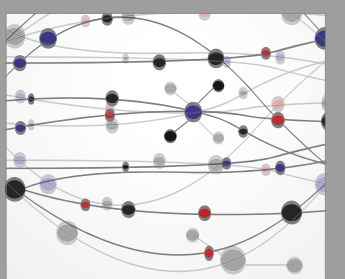

The Scientific World Journal

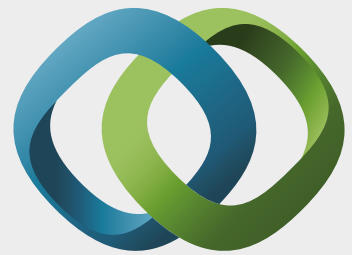

\section{Hindawi}

Submit your manuscripts at

https://www.hindawi.com
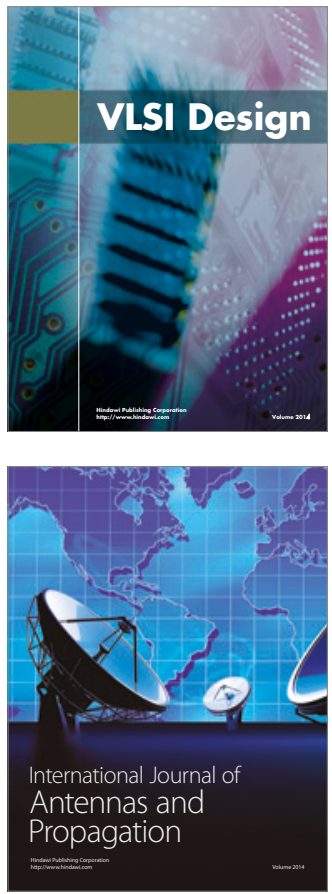

\section{Rotating}

Machinery
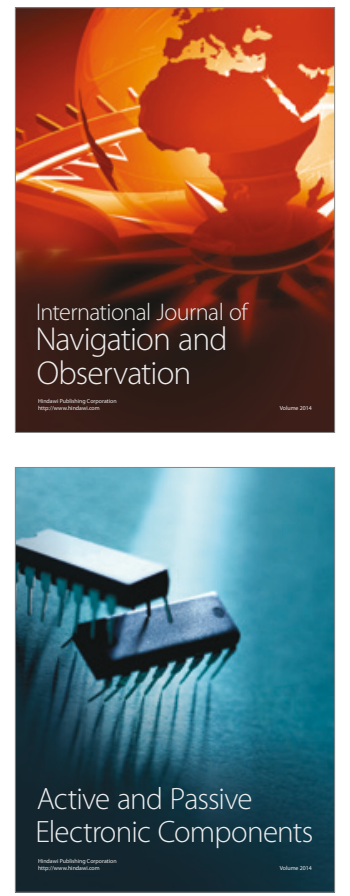
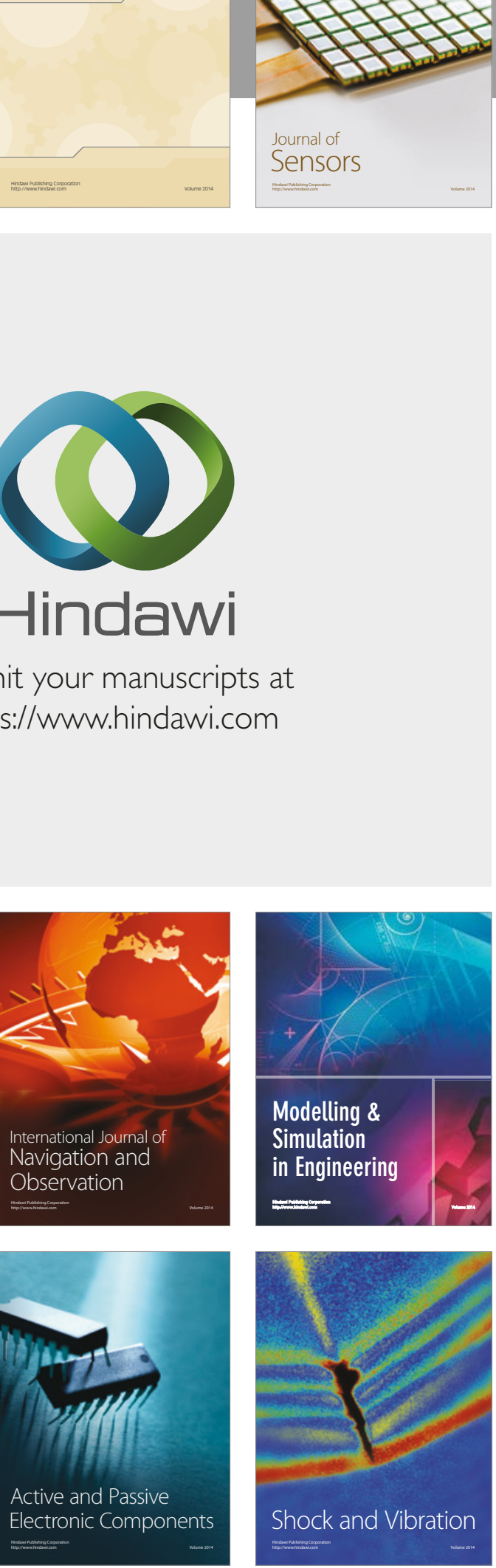
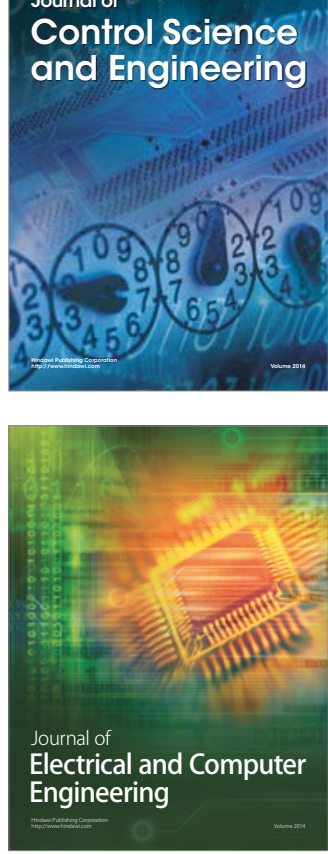

Distributed

Journal of

Control Science

and Engineering
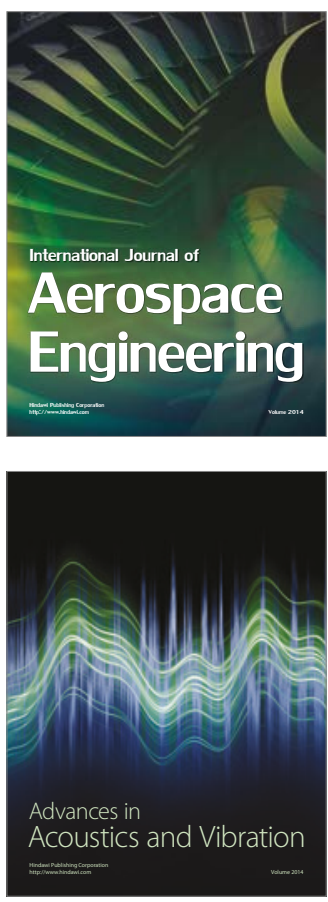

Sensor Networks 